\title{
Long-Term Driving Behaviour Modelling for Driver Identification
}

\author{
Letizia Marchegiani* and Ingmar Posner*
}

\begin{abstract}
Driver identification constitutes an important enabling technology in intelligent transportation systems, allowing the development and the use of in-car personalised functionalities and thwarting unauthorised usage. In this work, we leverage the literature in authentication tasks (e.g. speaker recognition) and present a framework for driver identification which employs Support Vector Machine (SVM) and Universal Background Model schemes. Our framework operates on accelerator and break pedal signals, and thus augments other technologies, such as microphones or cameras, if present. Moreover, our framework is compatible with vehicles which are limited to traditional sensing modalities. We evaluate the framework on 15 hours of driving data for a total of 416 $\mathrm{Km}$ travelled, comprising of messages from the CAN bus of an electric vehicle and GPS traces from four different drivers travelling on the same route, obtaining an accuracy of over $95 \%$ in the identification rate. Furthermore, our evaluation shows that UBM schemes outperform classification approaches traditionally adopted in driver identification literature by a significant margin.
\end{abstract}

\section{INTRODUCTION}

Driver identification represents an important aspect in the development of intelligent transportation system technologies, which aim to provide personalised in-car functionalities and respond to the threat of unauthorised users. Knowing the identity of the person driving ensures the vehicle's safety and allows for customisation according to the driver's preferences, improving comfort and efficiency in the use of the vehicle. In the case of electric cars, this efficiency can also be exploited in terms of more reliable estimates of the attainable range and predictions of the energy consumption (cf. [1], [2]). To guarantee a high level of portability and adaptability to different contexts and new drivers, the authentication process needs to take place in real time, transparently to the user and without necessarily involving traditional biometric measures, which would require the presence of specific sensors. Our goal is to augment other technologies, such as face or voice recognition, if present, with driving behaviour information, while providing vehicles, not equipped with additional sensing modalities, with a robust driver identification system. Building on previous studies which proved the effectiveness of pedal operation patterns as discriminant features for driver classification (e.g. [3], [4], and [5]), we develop a probabilistic framework for driver identification, which makes use of a spectral representation of accelerator and brake pedal signals (i.e. cepstral analysis), evaluating their discriminating power as sole features and when used in combination. More specifically, our system employs Support

\footnotetext{
* Authors are members of the Oxford Robotics Institute, University of Oxford, United Kingdom, \{letizia, ingmar\} arobots.ox.ac.uk
}

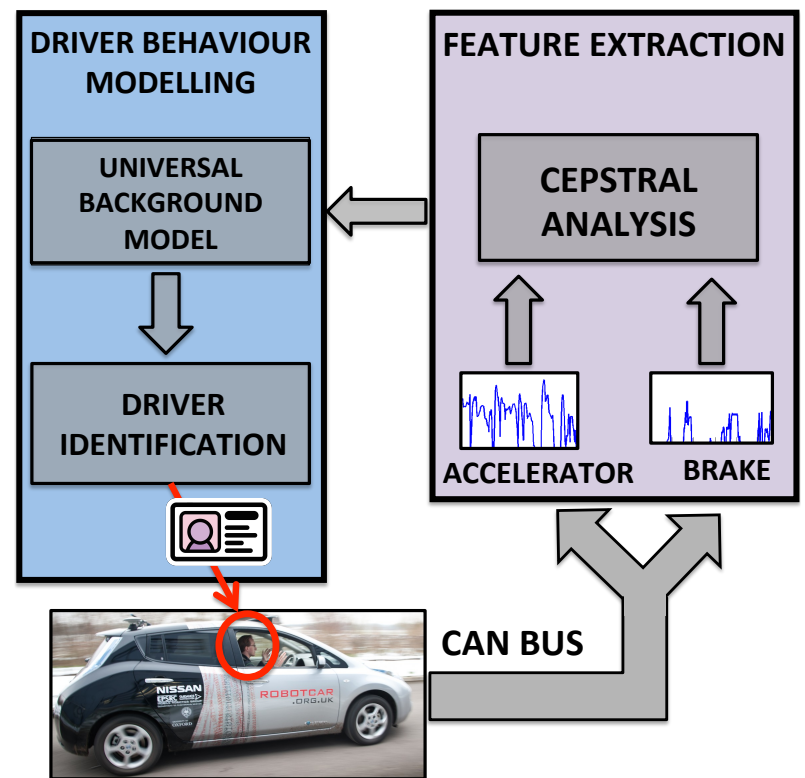

Fig. 1. Representation of the pipeline operating in the framework. Brake pedal and accelerator information is extracted from the CAN bus of the vehicle and cesptral analysis is performed to obtain the feature representation of the signals (purple area). Features are then fed to our driver behaviour model, which makes use of a Universal Background Model scheme, to authenticate the driver (blue area).

Vector Machines (SVMs) and a Universal Background Model (UBM) [6] paradigm. UBM has been extensively used in a variety of authentication tasks, such as speaker verification and recognition [7], [8], [9], online signature recognition [10], and periocular recognition [11], yielding remarkable performance. An illustration of our framework is provided in Figure 1.

Traditionally, UBMs rely on a large amount of data from different subjects, in order to represent subject-independent characteristics with respect to a model of subject-specific characteristics in authentication tasks [6]. In this work, we generate the UBM using data from several drivers to encapsulate the behaviour of the average driver against the target one. For this purpose, we collected 15 hours of driving data for a total of $416 \mathrm{Km}$ travelled, comprising of messages from the CAN bus of an electric vehicle and GPS traces from four different drivers travelling on the same route ${ }^{1}$. By collecting data on the same route and at similar times of the day (i.e. in similar traffic conditions), we aim to reduce as much as possible the effect of any environmental factor, while emphasising exclusively differences in the driving style. This dataset suits particularly well the scenario we want to

\footnotetext{
${ }^{1}$ The authors are planning to release the dataset.
} 
address, where identification is performed among a small group of drivers (i.e. a family sharing the same vehicle), with one of them being the main user of the car. In this scenario, indeed, the users of the car might be often driving along the same path. Furthermore, as the dataset covers a substantial time frame, our analysis can capture long-term characteristics in the driving style. With respect to previous works (e.g. [3], [4]) where identification systems were built and tested relying on data collected either in simulation or over few minutes long driving sessions, the nature of our dataset also allows us to evaluate our framework when operating over extensive periods of time. The results show that our system, despite the relative simplicity of the model adopted, is able to robustly authenticate the current user of the car. Lastly, we compare our framework against two classification methods with different complexity, traditionally utilised in driver identification literature, showing that the proposed UBM-based approach outperforms such methods by an appreciable margin, which increases with the number of drivers considered in the classification. To summarise, the main contributions of this paper are:

- the use of a UBM-based approach to perform driver identification, in contrast to previous literature which relies on classic classification frameworks;

- the use of a particularly challenging, and extensive, dataset, where driving data are collected along the same route and in similar traffic and driving conditions, which allows long-term analysis of the driver's behaviour in real-world scenarios.

The remainder of this article is structured as follows. Section II briefly describes related works. Section III illustrates the technical approach employed. Section IV presents the experimental evaluation of the framework. We conclude in Section V.

\section{RELATED WORKS}

Driver behaviour has been investigated in the literature from several perspectives and for different purposes. [12] performed the classification of certain driver characteristics, such as being cautious, average, expert, and reckless, for the development of an intelligent driver assistance system. A similar investigation has been carried out by [13] for humanin-the-loop automotive control architectures. This study also accounted for the driver mental state, the context or situation that the vehicle is in, and the surrounding environment. Anomalous behaviour detection and analysis have been explored in [14], [15]. Driver behaviour modelling has also played an important role in driver identification frameworks. Several features and their significance and impact in driver classification have been investigated in the literature. Most of these works make use of biometric information, equipping the car with cameras, microphones and other specific onboard sensors [16]. We envision our authentication system to augment these kinds of technologies with information pertaining the driving style, while also ensuring robust identification, in case of vehicles lacking such technologies. Previous studies in this direction mainly focused on pedal operation patterns, and on the use of Gaussian Mixture Models (GMM) and Neural Network (NN) frameworks to represent the driver's behaviour while performing specific tasks (e.g. [3], [4], and [5]). Other works [17] investigated the use of yaw rate sensors, steering angle information, and velocity measurements. Pedal operation patterns have been also used in [18] to build a model able to imitate the driver's operations and generate the desired throttle depression and braking pressure. The majority of these studies focus on predicting driver behaviour in specific circumstances for the development of intelligent driver advisory systems. Erdogan et al. [19] tested the combination of different classifiers, showing that signals like accelerator and brake pedal pressure actually carry biometric information able to provide the highest identification accuracy among a combination of different features, including steering angle, engine and vehicle speed. Building on the conclusions of [19], we propose a different approach to driving signal modelling, based on relatively simple SVM classifiers and UBM schemes, in order to authenticate the user of the vehicle. The obtained performance, indeed, suggests that, thanks to the use of the UBM paradigm, there is no real need of overloading the system with more complex machine learning frameworks, requiring additional computational resources. Furthermore, while previous studies have focused on the short-term behaviour of the driver, modelling either small fragments of driving data of about few minutes or driving responses collected in simulation, we perform an extensive data collection and build our models on several hours of driving data, which allows us to extend our analysis to long-term patterns and compartments in real-world scenarios. Lastly, as our dataset has been gathered along the same route and under similar weather and traffic conditions, external factors which might have a direct impact on the driving style without actually characterising it, are mitigated. This is especially useful in our scenario, as members of the same family (i.e. the target group of user we aim to identify) might be usually travelling the same path and the accuracy and the robustness of the systems might be affected by those environmental factors.

\section{METHODS}

The identification system presented in this work relies on the use of binary Support Vector Machines (SVM) [20] operating on acceleration and brake pedal information collected over several driving sessions for a total of $416 \mathrm{Km}$ travelled. We define a driving session as an entire lap of the route. For each of the driving sessions, the CAN messages containing information about the use of brake and accelerator signals are parsed and grouped into frames. More information about the feature representation of those signals is provided in Section III-B. For each driver, two different models, one for the brake pedal and one for the accelerator are built and used for identification. Specifically, we make use of the concept of Universal Background Model (UBM). UBMs are used to represent the expected alternative driving behaviour to be encountered during testing. More details about the UBM 


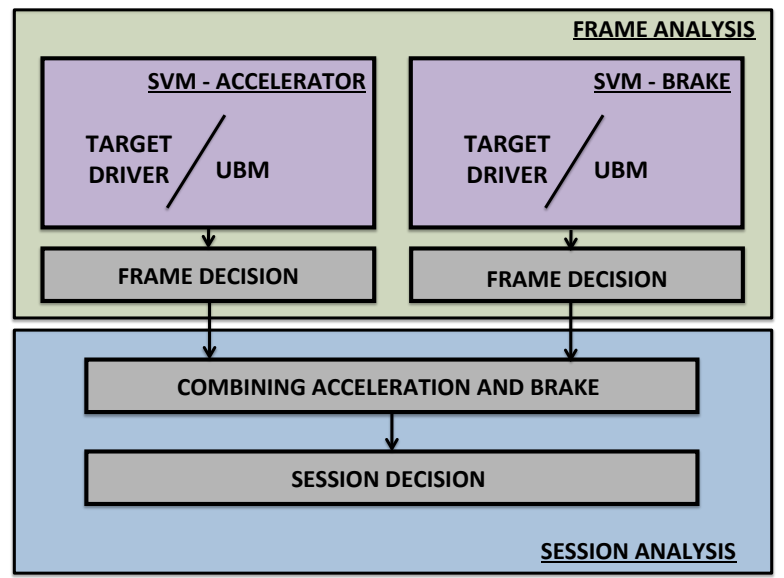

Fig. 2. Representation of the modelling strategy adopted. Two SVM models are built to discriminate between the target driver and the UBMs, relying on accelerator and brake information, respectively (purple boxes). Both SVMs operate on a per-frame basis, where a frame consists of 512 measurements, corresponding to about $5 \mathrm{~s}$ of driving, in case of the accelerator and $15 \mathrm{~s}$ for the brake information. (green area). For each of the feature, decisions made by the SVMs on each frame are merged following a majority voting strategy. A final decision referring to the entire driving session, i.e. an entire lap along the route (blue area), is then, obtained as a linear combination of the estimates provided by the accelerator and brake pedal majority voting methods. More details are provided in Section IV-D.

scheme are given in Section III-A. A summary of the all framework is given in Figure 2.

\section{A. The Universal Background Model}

The Universal Background Model has been successfully employed in several authentication tasks. Its use is based on the idea of building an alternative model to the one of the target subject to identify. The goal is to obtain a framework where the model of the target driver is compared against a model representing any potential alternative driver, where the latter is generated using a large amount of data from several different drivers, aiming to cover a great range of possible alternative patterns in feature space. This technique is close in spirit to one versus all (OVA) classifiers, where a multiclass problem is reduced to several binary problems. More specifically, when applying a OVA scheme, a binary classifier is built for each class, considering as positive samples the ones from that class and as negative the ones belonging to all the other classes. Despite its simplicity, previous works (e.g. [21]) have proved that OVA paradigms are particularly accurate when used in combination with properly tuned classifiers, such as SVMs. UBM is generally utilised together with Gaussian Mixture Models (GMMs), but literature also reports successful attempts (e.g. [22]) of using it with other classifiers, both in combination with GMMs and independently. Building on those premises, we propose the use of an SVM-UBM paradigm able to combine the representational power of the UBM with the discriminating power of SVMs, when operating in binary settings. Traditionally, it is possible to either rely on several UBMs tailored to specific subsets of drivers or a single UBM encapsulating the characteristics of all the potential population, which might be encountered at testing time. In this work, we chose to operate with a single UBM model, as our driver set is relatively small and we are not aiming to classify our drivers in subsets, according to specific driving traits. Concretely, to authenticate a target driver $D_{i}, i \in\{1,2,3,4\}$, we propose to train a UBM consisting of a combination of data from the remaining drivers $D_{j}, j \neq i$ in our dataset.

\section{B. Feature Representation}

As suggested by previous work [19], we employ as feature representation for our system CAN messages containing information on the accelerator position and the brake pedal pressure. In line with [3] and [5], we rely on a frequency representation of the pedal signals, based on cepstral coefficients and their first and second derivatives. Cepstral coefficients are especially useful to represent the envelope of the Fourier transform of a signal. More specifically, the complex cepstrum $x(t)$ of a sequence $s(t)$ is calculated by first computing the complex natural logarithm of the Fourier transform of $s(t)$, and then the inverse Fourier transform of the resulting sequence:

$$
x(t)=\mathcal{F}^{-1}[\ln (\mathcal{F}[s(t)])]
$$

The delta coefficients $\Delta(t)$, which refer to the first derivatives of the cepstrum coefficients are obtained by

$$
\Delta(t)=\frac{\sum_{n=1}^{N} n(x(t+n)-x(t-n))}{2 \sum_{n=1}^{N} n^{2}}, \text { with } N=2
$$

The delta-delta coefficients $\Delta \Delta(t)$, which refer to the second derivatives of the cepstrum coefficients are then obtained in a similar fashion from the delta coefficients. In total we use 39 coefficients between cepstrum, delta and delta-delta coefficients.

\section{EXPERIMENTS}

We carried out five experiments to evaluate our system. Through these experiments, we aim to investigate the performance of our UBM-based framework, as well as the discriminating power of both features (i.e. accelerator position and brake pedal pressure) when operating as sole features and when employed in combination. By varying the composition of the data used to train and test the models, we are interested in further analysing the robustness of the framework to different scenarios. In addition, we compare the behaviour of our framework against traditional classification approaches, typically employed in driver recognition tasks. A summary of the experimental evaluation is provided in Table I.

\section{A. The Data Set}

Our data set consists of 15 hours of driving data (for a total of $416 \mathrm{Km}$ travelled), comprising of messages from the CAN bus of an electric vehicle and GPS traces from four different drivers, travelling along the same route, shown in Figure 3. All driving sessions were carried out around the same time of the day in similar traffic conditions. These constraints, together with the choice of travelling on the same route, had the goal of reducing the impact of external and casual factors 


\begin{tabular}{ccccc}
\hline \multicolumn{5}{c}{ Experimental Evaluation } \\
\hline Experiment ID & Type & Training/Testing Configuration & Features & Frame/Session \\
\hline 1 & UBM-based Identification & Same Drivers in Training and Testing & A $\oplus$ B & Frame-based \\
2 & UBM-based Identification & Different Drivers in Training and Testing & A $\oplus$ B & Frame-based \\
3 & UBM-based Identification & Random Drivers in Training and Testing & A $\wedge$ B & Session-based \\
4 & Classification (Benchmark) & Binary SVM & A $\wedge$ B & Session-based \\
5 & Classification (Benchmark) & Gaussian Mixture Models (GMM) & A $\wedge$ B & Frame-based \\
\hline
\end{tabular}

TABLE I. Summary of the experimental evaluation performed. A and B indicate the accelerator position and the brake pedal pressure respectively. A session here refers to an entire lap along the route, while a frame indicates 512 measurements, corresponding to 5 s of driving, in case of the accelerator and $15 \mathrm{~s}$ for what concerns the brake related information.

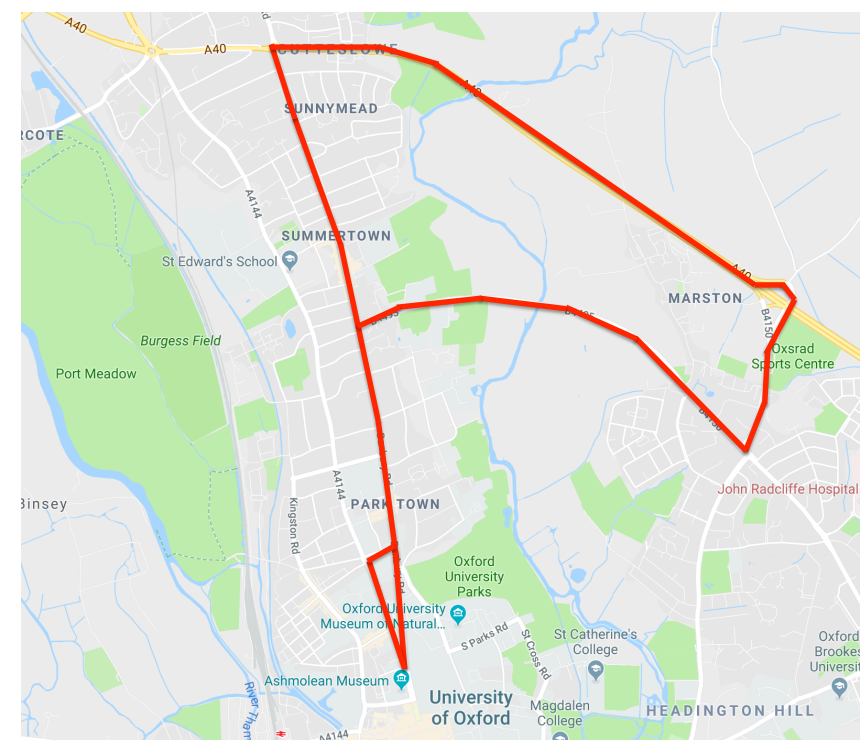

Fig. 3. Route travelled for the data collection. The route consists of different kinds of road types, such as highways, primary and secondary roads, as well as local roads. Such variety allows us to generalise our analysis of the driving style to different scenarios.

on the characterisation of the driving styles of the subjects. Yet, the route consists of different kinds of road types, such as highways, primary and secondary roads, as well as local roads. Such variety allows us to model the driving style in different environments. With respect to previous work, the specific composition of the dataset results to be more challenging for a driver identification system, hence enabling the evaluation of our framework in fully realistic scenarios, and over longer time frames. While driving, messages from the CAN bus of the vehicle have been recorded. The CAN messages carry information on the battery status, such as the instantaneous power consumption and remaining capacity, information on driving and car usage, such as steering angle and vehicle speed, and motor operation signals. In this work, we are interested in the accelerator position and the brake pedal pressure. Both signals are sampled at $90 \mathrm{~Hz}$ at a resolution of 10 bits. A complete description of the dataset and route characteristics are shown in Table II.

\begin{tabular}{cc}
\hline \multicolumn{2}{c}{ Dataset } \\
\hline Number of Drivers & 4 \\
Sessions per Driver & 8 \\
Route Length & $13 \mathrm{Km}$ \\
Total Distance Driven & $416 \mathrm{Km}$ \\
Total Driving Time & 15 hours \\
Total Number of Samples (A) & $\sim 4.10^{6}$ \\
Total Number of Samples (B) & $\sim 1.5 .10^{6}$ \\
Sampling Frequency & $90 \mathrm{~Hz}$ \\
Sample Resolution & $10 \mathrm{bits}$ \\
\hline
\end{tabular}

TABLE II. Dataset and Route characteristics. Total Number of Samples (A) refers to the total number of accelerator position samples. Total Number of Samples (B) refers to the total number of brake pressure samples. Samples where the car is not moving are omitted from the analysis. The brake pressure samples are fewer than the accelerator position ones, as drivers tend to use the brake pedal less frequently than the accelerator.

\section{B. Experiment 1: UBM with same drivers in training and testing}

The goal of this experiment is to explore the accuracy of the framework in successfully authenticating the user of the vehicle over short time frames, when only one of the two features (i.e. accelerator position and brake pedal pressure) is utilised (i.e. green area in Figure 2). Each frame consists of 512 measurements, corresponding to about $5 \mathrm{~s}$ of driving, in case of the accelerator and $15 \mathrm{~s}$ for the brake pedal information. The experiments are carried out in a leaveone-out cross validation framework. For each target driver $D_{i}, i \in\{1,2,3,4\}$, whose identity we aim to separately authenticate, we build two different SVM models: one for the acceleration and one for the brake information. Validation is carried out using seven of the eight driving sessions for training and the remaining one for testing. For the UBM, we use a random combination of sessions from the same randomly selected drivers $D_{j}, i \neq j$ both for training and testing. Figure 4 shows the Precision Recall curve for this experiment. Results suggest that, even when using only one of the two features and considering short time frames of few seconds of driving data, the system is able to accurately determine the identity of the driver. 


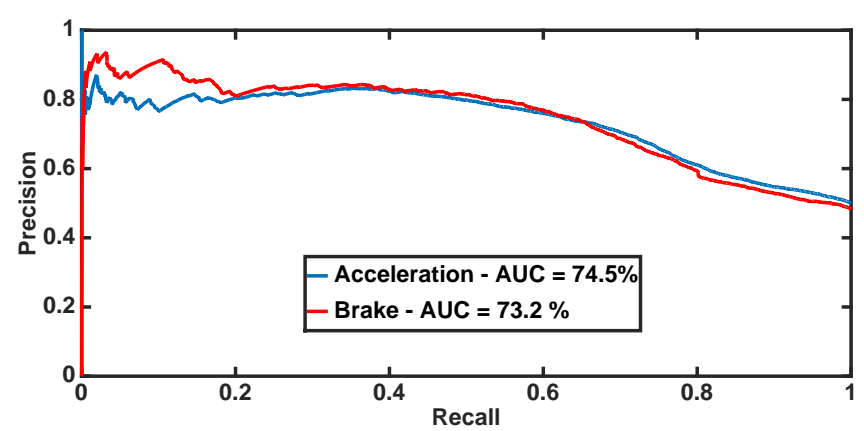

Fig. 4. Precision recall curve showing driver identification performance, in case of UBM with same drivers in training and testing. The blue line refers to the model based on the accelerator position information, while the red one refers to the model based on the brake pedal pressure information. $A U C=74.5 \%$ and $A U C=73.2 \%$ for the acceleration and brake model, respectively.

\section{Experiment 2: UBM with different drivers in training and testing}

The goal of this experiment is to investigate the robustness of the system to unseen driving patterns. With this purpose, unlike Experiment 1, the data chosen to build the UBM training set and the data used at testing time come from different drivers. Similarly to Experiment 1, the evaluation is carried out in a leave-one-out cross validation framework, on a per-frame basis, where each frame consists of 512 measurements. For each target driver $D_{i}$, with $i \in\{1,2,3,4\}$ whose identity we aim to separately authenticate, we build two SVM models: one for the acceleration and one for the brake information. In contrast to Experiment 1, for the UBM, we now use a random combination of sessions per driver $D_{j}, i \neq j$ for training and one (randomly picked from the remaining sessions of the remaining drivers $D_{j}, i \neq j$ ) for testing. Identification is carried out using seven of the eight driving sessions for training and the remaining for testing. Figure 5 shows the Precision Recall curve for this experiment. Results demonstrate that the performance of the

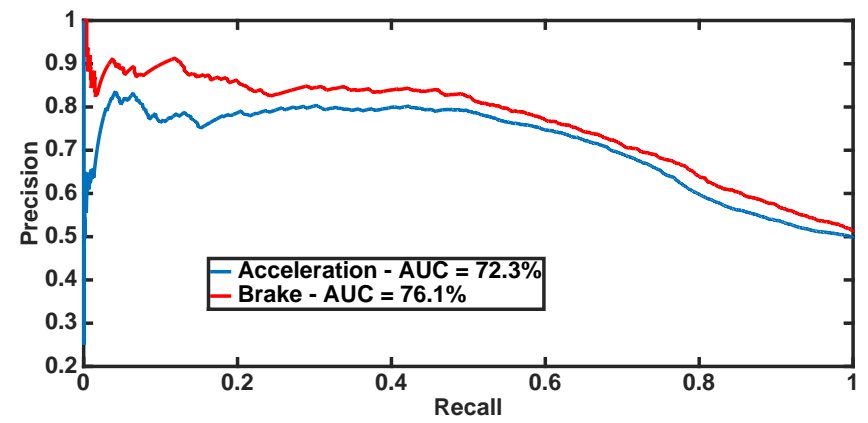

Fig. 5. Precision recall curve showing driver identification performance, in case of UBM with different drivers in training and testing. The blue line refers to the model based on the accelerator position information, while the red one refers to the model based on the brake pedal pressure information $A U C=72.3 \%$ and $A U C=76.1 \%$ for the acceleration and brake model, respectively.

framework is not affected by the different composition of the UBM and identification is robust, even when having in the testing phase data from drivers not employed to train the model, and even when using only one of the two features. We believe that this is due to use of the UBM paradigm which, as capable to fully exploit the nature of the dataset (i.e. collected over extensive driving sessions for a considerable amount of time), it is inherently able to capture the behaviour of the drivers in several circumstances as well the long-term patterns characterising it.

\section{Experiment 3: Combining Acceleration and Brake Infor- mation}

As it is not possible to combine brake and acceleration measurements belonging to the same frame to obtain a perframe prediction on the driver's identity (drivers accelerate and brake within the same frame only in rare cases), in this experiment, we evaluate the performance of the system on a per-session basis, relying on both features to make predictions. Following the framework structure illustrated in Figure 2 (blue area), estimates from the two models (acceleration and brake) are linearly combined to obtain a joint label for each session. More specifically, for each accelerator related frame $i$ in a specific session, we define the probability of the current driver $y_{i}$ being the target one $D$, according to the accelerator-based SVM, as $p\left(y_{i}^{*}=D \mid \mathbf{x}_{\mathbf{A}}^{*}\right)$, where $\mathbf{x}_{\mathbf{A}}^{*}$ refers to the cepstral feature representation of the accelerator pedal position sequence for that frame. Similarly, for each brake related frame $j$ we define the probability of the current driver $y_{j}^{*}$ being the target one $D$, according to the brake-based SVM, as $p\left(y_{j}^{*}=D \mid \mathbf{x}_{\mathbf{B}}^{*}\right)$, where $\mathbf{x}_{\mathbf{B}}^{*}$ refers to the cepstral feature representation of the brake pedal pressure sequence for that frame. The probability of the current driver $y^{*}$ being the target one $D$ for the entire sessions is then, obtained as:

$$
\begin{gathered}
p\left(y^{*}=D \mid \mathbf{x}_{\mathbf{A}}^{*}, \mathbf{x}_{\mathbf{B}}^{*}\right)=\alpha \frac{\mathrm{H}\left(\sum_{i=1}^{N^{A}} p\left(y_{i}^{*}=D \mid \mathbf{x}_{\mathbf{A}}^{*}\right)\right)}{N^{A}} \\
+(1-\alpha) \frac{\mathrm{H}\left(\sum_{j=1}^{N^{B}} p\left(y_{j}^{*}=D \mid \mathbf{x}_{\mathbf{B}}^{*}\right)\right)}{N^{B}}
\end{gathered}
$$

where $N^{A}$ and $N^{B}$ indicate the total number of acceleration and brake frames in the session, $\mathrm{H}$ is the Heaviside Function, and $\alpha: 0 \leq \alpha \leq 1$ is the parameter used to weight the contribution of the predictions of the two classifiers. The main idea is to first apply majority voting to the per-frame estimates provided by the accelerator and brake pedal SVMs and, finally, linearly combine these estimates to obtain a final decision referring to the entire driving session. The UBM is obtained using data from randomly selected drivers in training and testing. Figure 6 shows the precision recall curves obtained, averaged across the several sessions, in a leave-one-out cross validation framework, at varying of $\alpha$. We observe that the performance of the system improves with greater values of $\alpha$ (i.e. contribution of the accelerator information to the prediction is higher), reaching a maximum for $\alpha=0.8$. In addition, the results also show that the general performance of the system greatly improves when both features and all the frames in the session are used to make a decision. 


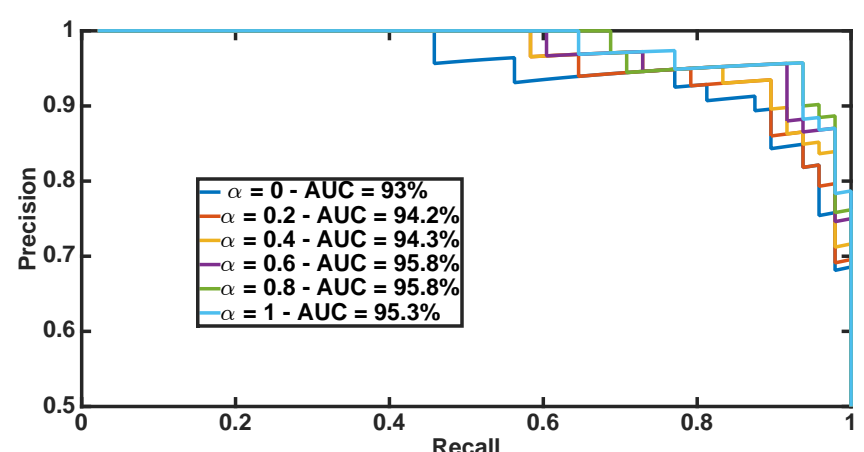

Fig. 6. Precision recall curve showing driver identification performance on a per-session basis, at varying of the parameter $\alpha$ (used to balance the contribution of the accelerator and brake models to the final decision.).

\section{E. Experiment 4: SVM Classification - Benchmark}

In the last two experiments, we compare the behaviour of the UBM-based approach proposed in this work against more traditional classification methods, generally employed in driving identification literature (e.g. [4] among others). In particular, the goal of Experiment 4 is to compare the behaviour of the identification system when using the UBM scheme and when, instead, relying on standard SVM binary classification (i.e. one against one). Also in this case, the experiments are carried out in leave-one-out cross validation framework. For each pair of target drivers we obtain an SVM classifier using seven of the eight driving sessions for training and the remaining one for testing. No UBM is employed in this evaluation. Two different models are again built: one to represent the accelerator position and the other to represent the brake pedal pressure. Predictions are then combined in a similar fashion as in Experiments 3 (cf. Section IV-D). Figure

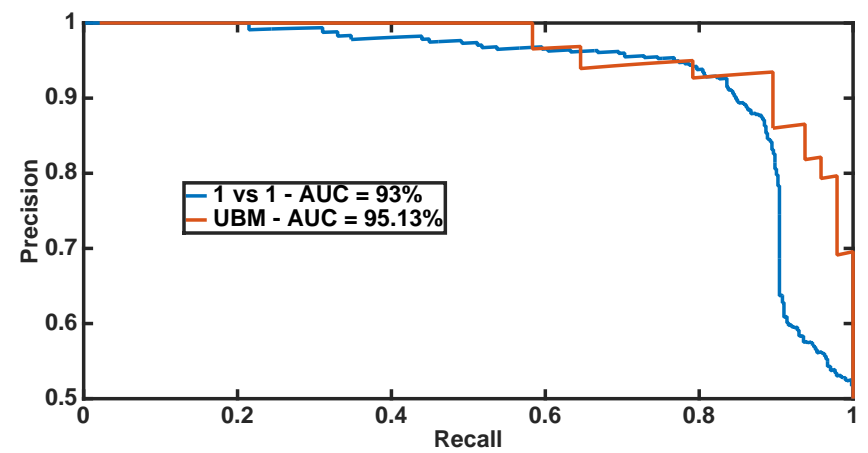

Fig. 7. Precision recall curves showing driver classification performance on a per-session basis, obtained with $\alpha=0.8$, as providing the best performance according to the evaluation carried out for Experiments 3. In this experiment, no UBM is employed, but standard binary classification on pairs of drivers (one against one). For direct comparison, the figure also reports the precision recall curve when the UBM scheme is used, for $\alpha=0.8$ (cf. Figure 6)

7 reports the precision recall curves for this experiment. The curve is computed with $\alpha=0.8$, as providing the best performance according to the evaluation carried out for Experiments 3. For direct comparison, the figure also reports the precision recall curve when the UBM scheme is used, for $\alpha=0.8$ (cf. Figure 6). We observe that the
UBM paradigm evaluated in Experiment 3 outperforms the traditional classification system utilised in this experiment.

\section{F. Experiment 5: GMM Classification - Benchmark}

Lastly, we compare our framework against a classification system relying on the use of Gaussian Mixture Models. Following previous works (e.g. [4], [3]), for each driver $D_{i}$ with $i \in\{1,2,3,4\}$ a model $\Theta_{A}^{i}$ and a model $\Theta_{B}^{i}$ are built to represent the accelerator and the brake signal patterns. Classification is performed on a per-frame basis, where the whole dataset is partitioned into training and testing sets, and no notion of session is present. In this case, $85 \%$ of the data is used for training and $15 \%$ for testing. At testing phase, the current driver is identified as the one yielding the maximum weighted GMM log likelihood over the sequence of cepstral feature vectors for the accelerator and brake pedals. Figure 8 shows the results of the classification, when using 16 Gaussian models in the mixture. We report the results for a mixture with 16 Gaussian models, as it is the configuration providing the best performance. In particular, the figure reports the average identification rate and one standard deviation on error bars, at varying of the parameter $\alpha$ (used to balance the contribution of the accelerator and brake models to the final decision, similarly to Experiments 3 and 4). Standard deviation reported on the error bars is computed over 1000 random partitions of the dataset into training and testing sets.

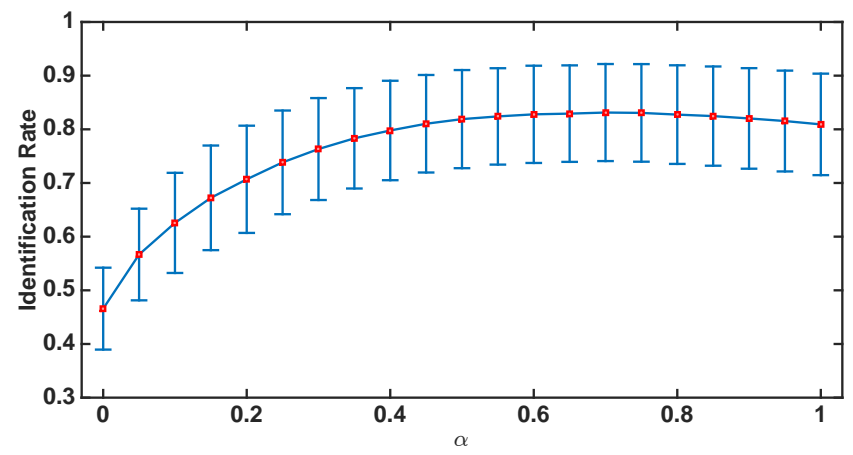

Fig. 8. Figure reports average recognition rate and one standard deviation on error bars, at varying of the parameter $\alpha$ (used to balance the contribution of the accelerator and brake models to the final decision.), when using a Gaussian Mixture Model with 16 Gaussian models in the mixtures.

We observe that the performance of the system increases with higher values of $\alpha$ reaching the greatest accuracy (average identification rate 83\%) when $\alpha=0.7$. The UBMbased system outperforms the GMM by a significant margin for all values of $\alpha$, and this difference is considerably higher for smaller values of $\alpha$, when the impact of the brake pedal information is prominent. This is due to the fact that less data related to the brake pedal signals is available, as drivers tend to use the brake pedal much less frequently than the accelerator. These results are in line with previous works, and highlight the role played by the UBM scheme, which allows to better capture the behaviour of a specific driver, even when less information is available, thanks to the way the impostor model is built. 
In summary, we conclude that, for this task, the UBMbased approach proposed outperforms more traditional classification approaches by an appreciable margin and that this margin increases with the number of drivers considered in the classification.

\section{CONCLUSION}

We presented a framework for driver identification, which makes use of Support Vector Machines and a Universal Background Model paradigm. We evaluate the framework on $416 \mathrm{Km}$ of driving data, comprising of messages from the CAN bus of an electric vehicle and GPS traces from four different drivers travelling on the same route, obtaining great accuracy in the authentication. Evaluation also suggested that for this task the UBM schemes are more robust and accurate compared to standard classification approaches, traditionally used in driving identification literature. This difference in the performance is especially apparent when less data is available and when multi-class methods are applied. Future work could investigate the effect of different kinds of road (i.e. highways, local streets, etc.), as well as the driver's status, on driving behaviour signals and driver identification. Lastly, the effect of missing data [23] on identification performance could be explored.

\section{ACKNOWLEDGMENT}

This work was supported by the UK EPSRC Programme Grant EP/M019918/1. The authors would like to thank the drivers for their help in the data collection.

\section{REFERENCES}

[1] P. Ondruska and I. Posner, "The route not taken: Driver-centric estimation of electric vehicle range," in Twenty-Fourth International Conference on Automated Planning and Scheduling, 2014.

[2] — , "Probabilistic attainability maps: Efficiently predicting driverspecific electric vehicle range," in Intelligent Vehicles Symposium Proceedings, 2014 IEEE. IEEE, 2014, pp. 1169-1174.

[3] T. Wakita, K. Ozawa, C. Miyajima, K. Igarashi, I. Katunobu, K. Takeda, and F. Itakura, "Driver identification using driving behavior signals," IEICE TRANSACTIONS on Information and Systems, vol. 89, no. 3, pp. 1188-1194, 2006.

[4] C. Miyajima, Y. Nishiwaki, K. Ozawa, T. Wakita, K. Itou, and K. Takeda, "Cepstral analysis of driving behavioral signals for driver identification," in Acoustics, Speech and Signal Processing, 2006. ICASSP 2006 Proceedings. 2006 IEEE International Conference on, vol. 5. IEEE, 2006, pp. V-V.

[5] A. Wahab, T. C. Keong, H. Abut, and K. Takeda, "Driver recognition system using fnn and statistical methods," in Advances for In-Vehicle and Mobile Systems. Springer, 2007, pp. 11-23.

[6] D. Reynolds, "Universal background models," Encyclopedia of biometrics, pp. 1547-1550, 2015.

[7] D. A. Reynolds, T. F. Quatieri, and R. B. Dunn, "Speaker verification using adapted gaussian mixture models," Digital signal processing, vol. 10 , no. $1-3$, pp. 19-41, 2000.

[8] T. May, S. Van De Par, and A. Kohlrausch, "Noise-robust speaker recognition combining missing data techniques and universal background modeling," IEEE Transactions on Audio, Speech, and Language Processing, vol. 20, no. 1, pp. 108-121, 2012.

[9] L. Marchegiani, F. Pirri, and M. Pizzoli, "Multimodal speaker recognition in a conversation scenario," in International Conference on Computer Vision Systems. Springer, 2009, pp. 11-20.

[10] E. A. Rua, E. Maiorana, J. L. A. Castro, and P. Campisi, "Biometric template protection using universal background models: An application to online signature," IEEE Transactions on Information Forensics and Security, vol. 7, no. 1, pp. 269-282, 2012.
[11] J. C. Monteiro and J. S. Cardoso, "Periocular recognition under unconstrained settings with universal background models." in BIOSIGNALS, 2015 , pp. 38-48.

[12] N. Lin, C. Zong, M. Tomizuka, P. Song, Z. Zhang, and G. Li, "An overview on study of identification of driver behavior characteristics for automotive control," Mathematical Problems in Engineering, vol. 2014, 2014.

[13] K. Driggs-Campbell, V. Shia, and R. Bajcsy, "Improved driver modeling for human-in-the-loop vehicular control," in Robotics and Automation (ICRA), 2015 IEEE International Conference on. IEEE, 2015, pp. 1654-1661.

[14] K. D. Kusano and H. C. Gabler, "Identification of target populations for current active safety systems using driver behavior," in Intelligent Vehicles Symposium (IV), 2012 IEEE. IEEE, 2012, pp. 655-660.

[15] W. Wang, Q. Cao, K. Ikeuchi, and H. Bubb, "Reliability and safety analysis methodology for identification of drivers erroneous actions," International Journal of Automotive Technology, vol. 11, no. 6, pp. 873-881, 2010.

[16] A. Riener and A. Ferscha, "Supporting implicit human-to-vehicle interaction: Driver identification from sitting postures," in The First Annual International Symposium on Vehicular Computing Systems (ISVCS 2008), 2008, p. 10.

[17] H. Tokutake, Y. Sugimoto, and T. Shirakata, "Real-time identification method of driver model with steering manipulation," Vehicle System Dynamics, vol. 51, no. 1, pp. 109-121, 2013.

[18] J. Wang, L. Zhang, D. Zhang, and K. Li, "An adaptive longitudinal driving assistance system based on driver characteristics," Intelligent Transportation Systems, IEEE Transactions on, vol. 14, no. 1, pp. 1$12,2013$.

[19] H. Erdogan, A. Ereil, and H. Abut, "Experiments on decision fusion for driver recognition," in Advances for In-Vehicle and Mobile Systems. Springer, 2007, pp. 1-9.

[20] V. Vapnik, The nature of statistical learning theory. Springer Science \& Business Media, 2013.

[21] R. Rifkin and A. Klautau, "In defense of one-vs-all classification," Journal of machine learning research, vol. 5, no. Jan, pp. 101-141, 2004.

[22] M. Liu, B. Dai, Y. Xie, and Z. Yao, "Improved gmm-ubm/svm for speaker verification," in Acoustics, Speech and Signal Processing, 2006. ICASSP 2006 Proceedings. 2006 IEEE International Conference on, vol. 1. IEEE, 2006, pp. I-I.

[23] S. G. Karadogan, L. Marchegiani, L. K. Hansen, and J. Larsen, "How efficient is estimation with missing data?" in Acoustics, Speech and Signal Processing (ICASSP), 2011 IEEE International Conference on. IEEE, 2011, pp. 2260-2263. 